\title{
Analysis of beta-carotene hydroxylase gene cDNA isolated from the American oil-palm (Elaeis oleifera) mesocarp tissue cDNA library
}

\author{
Subhash J. Bhore ${ }^{1,2 *}$, Amelia Kassim ${ }^{1}$, Chye Ying Loh ${ }^{2}$, Farida H. Shah ${ }^{1}$ \\ ${ }^{1}$ Molecular Biology Division, Melaka Institute of Biotechnology, Lot 7, Melaka International Trade Centre City, 75450 Ayer Keroh, Melaka, Malaysia; \\ ${ }^{2}$ Department of Biotechnology, Faculty of Applied Sciences, AIMST University, Bedong-Semeling Road, Bedong, 08100, Kedah Darul Aman, Malaysia; \\ Subhash J Bhore- Email: subhashbhore@gmail.com; Phone:+60-4-429 8176; Fax: +60-4-429 8109, "Corresponding author
}

Received August 19, 2010; Accepted August 26, 2010; Published September 20, 2010

\begin{abstract}
It is well known that the nutritional quality of the American oil-palm (Elaeis oleifera) mesocarp oil is superior to that of African oil-palm (Elaeis guineensis Jacq. Tenera) mesocarp oil. Therefore, it is of important to identify the genetic features for its superior value. This could be achieved through the genome sequencing of the oil-palm. However, the genome sequence is not available in the public domain due to commercial secrecy. Hence, we constructed a cDNA library and generated expressed sequence tags $(3,205)$ from the mesocarp tissue of the American oil-palm. We continued to annotate each of these cDNAs after submitting to GenBank/DDBJ/EMBL. A rough analysis turned our attention to the beta-carotene hydroxylase (Chyb) enzyme encoding cDNA. Then, we completed the full sequencing of cDNA clone for its both strands using M13 forward and reverse primers. The full nucleotide and protein sequence was further analyzed and annotated using various Bioinformatics tools. The analysis results showed the presence of fatty acid hydroxylase superfamily domain in the protein sequence. The multiple sequence alignment of selected Chyb amino acid sequences from other plant species and algal members with E. oleifera Chyb using ClustalW and its phylogenetic analysis suggest that Chyb from monocotyledonous plant species, Lilium hubrid, Crocus sativus and Zea mays are the most evolutionary related with E. oleifera Chyb. This study reports the annotation of E. oleifera Chyb.
\end{abstract}

Keywords: African oil-palm, American oil-palm, fatty acids, fatty acid hydroxylase, oleic acid, sterol desaturase, zeaxanthin

Abbreviations: ESTs, expressed sequence tags; EoChyb, Elaeis oleifera beta-carotene hydroxylase; MC, main cluster

\section{Background:}

Oil-palm is the major commodity worldwide. The second largest source of fats and oils to the world market of fats and oils is from Elaeis guineensis Jacq Tenera, which is commonly known as African oil-palm. Malaysia is the main producer and exporter of the palm oil. African oil-palm is cultivated on industrial scale due to high palm oil yield derived from its fruit mesocarp tissue. African oil-palm, E. guineensis Jacq have three different forms (also called as varieties), namely, 'Pisifera', 'Dura', and 'Tenera'. These three forms are distinguished on the basis of shell thickness of fruits. The commercially cultivated African oil-palm is a hybrid from 'Dura' (ㅇ) and 'Pisifera' ( $)$ [1]. Another oil-palm species which is economically less important is Elaeis oleifera. This species is also called as American oil-palm.

Palmitic acid, $\left(\mathrm{C}_{16: 0}\right)$ is the predominant fatty acid in palm oil derived from E. guineensis Jacq. Tenera fruit mesocarp tissue. Whereas oil derived from E. oleifera fruit mesocarp tissue is predominant with oleic acid $\left(\mathrm{C}_{18: 1}\right)$ (68.6\%), a fatty acid good for health [2]. The $\mathrm{C}_{16: 0}$ is the major $(44 \%)$ saturated fatty acid in palm oil derived from E. guineensis. However, the $\mathrm{C}_{16: 0}$ content in E. oleifera is only $25 \%$. In spite of the high content of the healthy $\mathrm{C}_{18: 1}$ in the E. oleifera oil, it is not preferred for commercial plantation due to its poor oil yield.

The single pass, partial sequencing of randomly isolated anonymous cDNA clones also called as ESTs has become a rapid and cost-effective means in gaining information about gene expression and their regulation [3]. In addition, generated ESTs data is useful in new and novel gene's discovery [4], evaluation of the genome for gene content and its structure, ISSN 0973-2063 (online) 0973-8894 (print) Bioinformation 5(3): 104-112 (2010) and for in silico comparative expression analysis between different plant tissues [5]. Most importantly, ESTs serves as a valuable resource for highthroughput expression analysis using cDNA-microarray technology [6]. In higher plants, numerous genes have been identified by random nucleotide sequencing of cDNA clones [7-11]. Therefore, in order to study the gene expression and their patterns in E. oleifera fruit mesocarp tissue, ESTs generation project was initiated. So far, 3,205 ESTs are generated from 17 weeks old E. oleifera mesocarp tissue cDNA library (our unpublished work). Beta carotene hydroxylase $(C h y b)$ is one of the isolated cDNA (ESTs) clones.

By understating potential applications of Chyb in genetic engineering of oil-palm and or in other plants, clone was fully sequenced. The Chyb is involved in zeaxanthin biosynthesis by hydroxylating beta-carotene, but the enzyme may be involved in other pathways [12-13]. The products of this enzyme are zeaxanthin and beta-cryptoxanthin [14]. Beta-carotene, zeaxanthin and beta-cryptoxanthin are categorized under carotenoids which have many industrial applications as food and feed additives, and are used in cosmetics and as nutraceutical. Currently, vitamin A deficiency is a global health burden which could be alleviated through provitamin A carotenoid biofortification in suitable plants [15]. If we understand in depth the regulation of carotenoid biosynthesis, enhancement of beta-carotene could be done by limiting beta-carotene hydroxylation. Therefore in order to understand more about EoChyb, its cDNA clone is analyzed using computational tools. The nucleotide and protein sequence of EoChyb cDNA is analyzed and annotated in this study to find out their features. The EoChyb annotation and features are reported in this paper. 


\begin{abstract}
Methodology:
cDNA library and EoChyb clone isolation

Elaeis oleifera, seventeen week's old [weeks after anthesis (WAA)] mesocarp tissue cDNA library constructed using the 'CloneMiner cDNA library construction kit' (Invitrogen Corporation) for ESTs generation (our unpublished work) was used to isolate EoChyb. The EoChyb cDNA clone was isolated by random method of cDNA isolation [3].
\end{abstract}

The cDNA nucleotide sequence reported in this paper has been submitted to GenBank/DDBJ/EMBL under accession number EU057623.

\section{Nucleotide sequencing}

The E. coli DH5 $\alpha$ cells harboring EoChyb cDNA were cultivated in $10 \mathrm{ml}$ LB medium (supplemented with Kanamycin) overnight in dark at $37{ }^{\circ} \mathrm{C}$, $160 \mathrm{rpm}$. The cultivated $E$. coli DH5 $\alpha$ cells were harvested from broth and plasmid DNA was isolated by using a commercial kit, Wizard ${ }^{\circledR}$ Plus SV Minipreps DNA purification system (Promega). Sequencing reactions were carried out for both strands using M13 (Forward); 5'GTAAAACGACGGCCAG-3' and M13 (Reverse); 5' GGATAACAATTTCACACAGG-3' primers.

\section{cDNA and protein sequence analysis}

The cDNA sequence was edited manually to eliminate vector and adaptor sequences from 5' and 3' ends. Finalized cDNA sequence analysis was performed using online free bioinformatics tools. The similarity searches were performed using blast programs (BlastN, BlastX, and BlastP) against the databases available at NCBI [16]. EMBOSS pairwise alignment algorithm [http://www.ebi.ac.uk/Tools/emboss/align/] was used to compare 2 cDNA sequences to find out homology $\%$. To find out general features of the cDNA sequence including amino acid composition and isoelectric point analysis was performed online using bioinformatics tools available at JustBio [17]. Guanine and cytosine content (GC \%) calculation was carried out by using 'DNA/RNA base composition calculator', a free online bioinformatics tool that calculates the molecular mass, elemental composition, base composition, and percent AT and GC content for cDNA/DNA/RNA sequences [18]. Alignment of amino acid sequences and dendrogram construction was carried out using multiple sequence alignment by ClustalW [19] program. Whereas, to find out fully conserved residues; residues with conserved strong groups and residues with conserved weak groups in EoChyb, amino acid sequences were aligned by using clustal 2.0.11 multiple sequence alignment program.

\section{Results:}

\section{EoChyb clone isolation}

By random method of cDNA clone isolation, EoChyb clone was isolated from 17 day old mesocarp tissue cDNA library. The serial number of this randomly isolated cDNA clone in ESTs generation project was 2962; hence clone identity 'EoEST-2962' was given to EoChyb cDNA clone.

\section{Nucleotide sequencing}

The +ve and -ve strand of EoChyb cDNA clone were sequenced using M13 forward and M13 reverse primers, respectively. The EoChyb +ve and -ve strand cDNA sequence after removal of vector and adaptor sequence was compared and searched for overlaps using blast (bl2seq) program [16]. The results produced shows that EoChyb cDNA sequence is $1414 \mathrm{bp}$ in length. By analyzing the finalized cDNA sequence and its deduced amino acid sequence, Chyb identity was given to the cDNA clone.

\section{cDNA and protein sequence analysis}

The general features of the cDNA nucleotide and protein sequence as revealed by bioinformatics tools available at JustBio are summarized in Table 1 (see supplementary material). The annotated nucleotide sequence was submitted to GenBank/DDBJ/EMBL under accession number EU057623. Whereas the comparison of the EoChyb at nucleotide and protein level with Chyb from other plant species and algal members is depicted in Table 2 (see supplementary material). The nucleotide and deduced amino acid sequences of EoChyb cDNA, an open reading frame (ORF), 5' and 3' untranslated regions (UTR), initiation and termination codon, and the amino acid residues of beta-carotene hydroxylase and sterol desaturase conserved domains are shown in Figure 1. The EoChyb, and Chyb from other selected plants and algal members Chyb amino acid sequence alignment based phylogenetic analysis was carried out and the constructed dendrogram is shown in Figure 2. Clustal 2.0.11 multiple sequence alignment program produced results of EoChyb amino acid sequence alignment with amino acid sequences of Chyb from other organisms and showed single fully conserved residues, residues with conserved strong groups and residues with conserved weak groups (Figure 3).

\section{Discussion:}

The availability of EoChyb cDNA clone in 17 day old mesocarp tissue cDNA library indicates that Chyb is expressed in developing E. oleifera fruit mesocarp tissue. However, the level of its expression, pattern of expression and tissue specificity is not known. The analysis of developing fruit mesocarp by Northern blot technique could shade the light on level and pattern of its expression [20]. The GC content in EoChyb cDNA is $56 \%$. This GC \% is close to the predicted GC content in coding sequences of Elaeis oleifera and Elaeis guineensis [21-22]. Homology analysis using BlastP indicates that EoChyb protein shows 82-98\% homology with Chyb from other monocot plant species. Comparison of EoChyb with Chyb from 13 dicot plant species shows range of homology from 71-98\%. However, Chyb from algal member shows only $78-84 \%$ homology with EoChyb (Table 2, see supplementary material). The relatively low level of EoChyb homology with Chyb from algal member is in line with the evolution in plant species. Monocots are highly evolved in comparison with the dicots and algae. Conserved domain search in EoChyb indicates that amino acid residue 46-324 are part of the beta-carotene hydroxylase (fatty acid hydroxylase super family) conserved domain; and amino acid residues 184-309 belongs to the sterol desaturase [lipid metabolism] domain (Figure 1). However, latter's role in sterol desaturation is not clear [23]. The EoChyb amino acid composition analysis revealed that it is rich in alanine (A) amino acid (See Figure $\mathbf{1}$ and Table $\mathbf{3}$ in Supplementary material).

The dendrogram constructed to study the phylogenetic relationship between EoChyb and Chyb protein sequences from 13 dicots, 3 monocots and 3 algal members shows $3 \mathrm{MC}$. The Chyb proteins from dicots, monocots and algal members were precisely grouped in $\mathrm{MC} 1, \mathrm{MC} 2$, and MC3 respectively (Figure 2). All the Chyb proteins from dicotyledonous plants in MC1 were sub-grouped into 3 sub-clusters. The sub-cluster-1 is of Chyb from Citrus sinensis and Glycine max. The sub-cluster-2 is of Chyb from Coffea arabica, Solanum lycopersicum, Gentiana lutea, Diospyros kaki and Capsicum annuum. Whereas, Vitis vinifera, Adonis aestivalis, Chrysanthemum $x$ morifolium, Brassica napus, Arabidopsis thaliana and Daucus carota were grouped under sub-cluster- 3 of MC1. All the Chyb protein sequences from monocots, Crocus sativus, Elaeis oleifera, Lilium hybrid and Zea mays used in phylogenetic analysis were grouped under $\mathrm{MC2}$; and three algal Chyb protein sequences from Chlamydomonas reinhardtii, Muriella zofingiensis and Haematococcus pluvialis were grouped under MC3 (Figure 2). This classification of Chyb is in line with the phylogenetic lineages that have molecular data in the NCBI databases [24]. The dendrogram analysis also reflects that EoChyb is phylogenetically closer to the Chyb from Lilium hybrid and other monocots used in the study.

The multiple sequence alignment of Chyb amino acid sequences of EoChyb and Chyb protein sequences from 13 dicots, 3 monocots and 3 algal members (Table 2 see supplementary material) clearly shows the well conserved catalytic residues in $C h y b$. The analysis of the multiple sequence alignment of Chyb amino acid sequences clearly shows that in total 51 amino acid residues are fully conserved in Chyb of the all plant species and algal members (Figure 3). It was also evident that across the Chyb amino acid sequence, in total 28 amino acid residues were conserved strongly (Figure 3). 


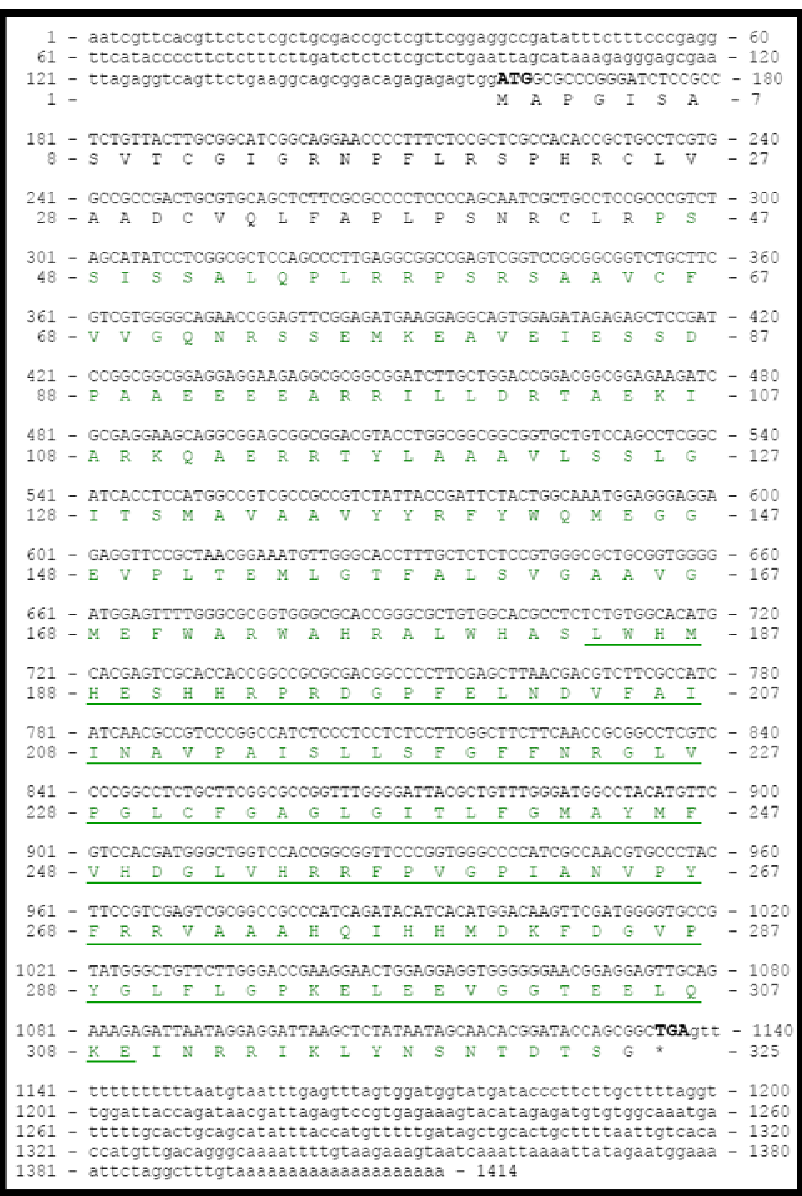

Figure 1: Nucleotide and deduced amino acid sequences of E. oleifera beta-carotene hydroxylase cDNA clone. An open reading frame and noncoding regions are shown in capital and small letters, respectively. The deduced amino acid sequence is given below the nucleotide sequence, which is numbered at the both ends of each sequence line. The open reading frame encodes for a protein of 325 amino acid residues. Amino acid residues are numbered beginning with the initial methionine till last glycine $(\mathrm{G})$ residue. Initiation and termination codons are shown in bold. The beta-carotene hydroxylase (fatty acid hydroxylase super family) conserved domain (PLN02601) [25] residues are shown in green colour. The sterol desaturase [Lipid metabolism] domain (ERG3) [26] residues are underlined. *represent the termination codon. This cDNA was isolated by random method of gene isolation from $E$. oleifera 17 week old mesocarp tissue cDNA library.

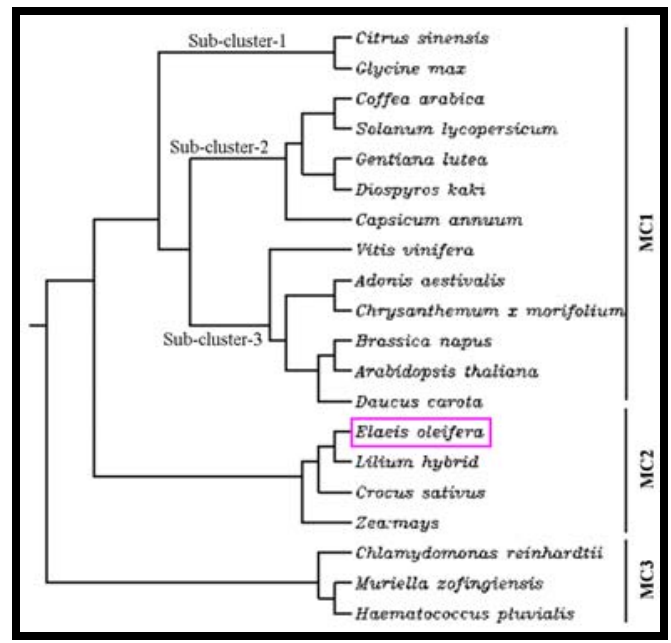

Figure 2: Rooted dendrogram showing clustering of beta-carotene hydroxylase (Chyb) from E. oleifera and other organisms. Amino acid sequences for different organisms were obtained from NCBI database. Alignment of amino acid sequences and dendrogram construction was carried out using multiple sequence alignment by ClustalW [19] program using default parameters. Location of E. oleifera Chyb in phylogenetic tree is shown in pink box. The ID of Chyb proteins used in the study is given in Table 2 (see supplementary material). MC stands for main cluster.

ISSN 0973-2063 (online) 0973-8894 (print) 106

Bioinformation 5(3): 104-112 (2010) 


\section{Bioinformation}

\section{Volume 5}

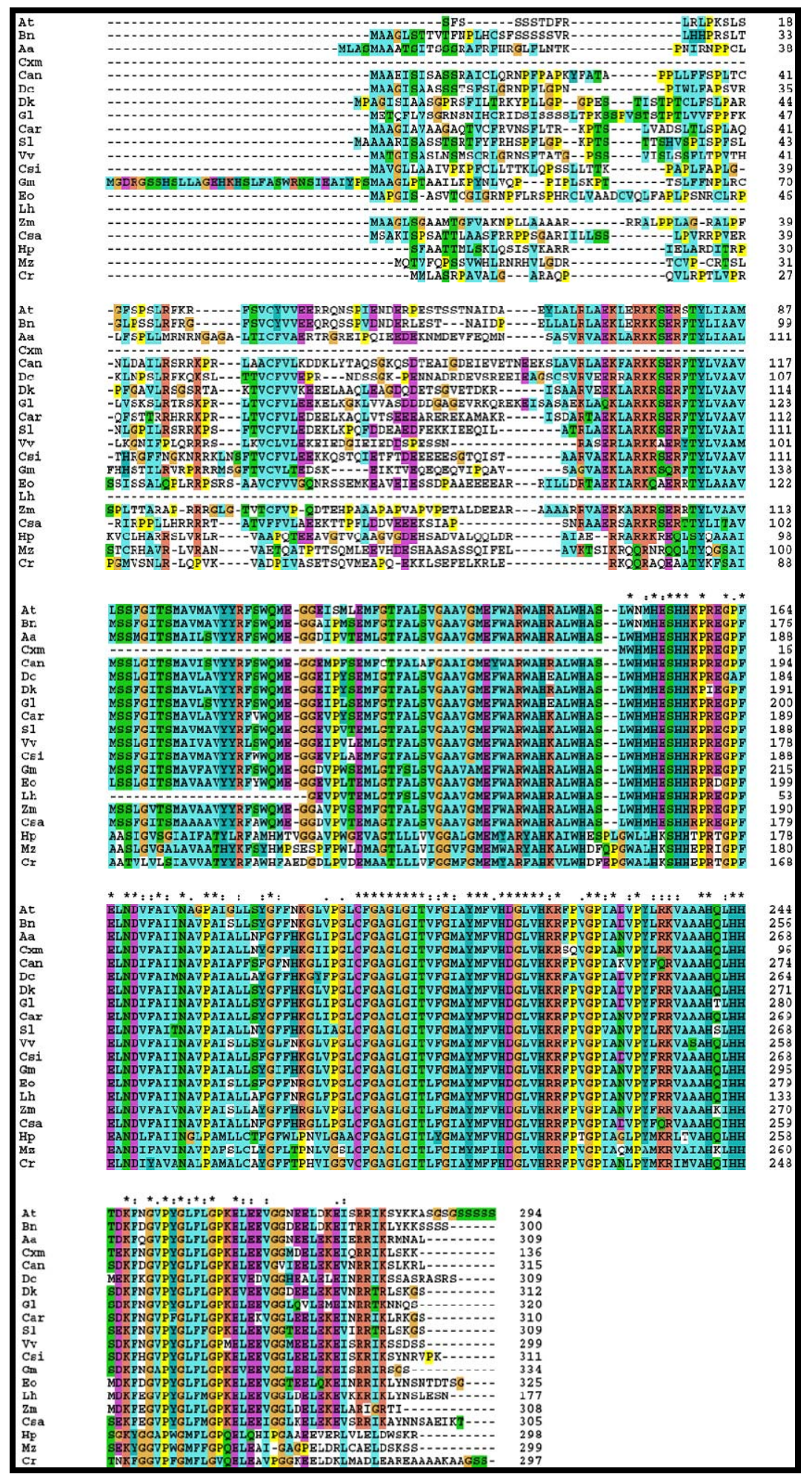

Figure 3: Similarity comparison of amino acid sequences of the E. oleifera beta-carotene hydroxylase (Chyb) protein and Chyb amino acid sequences from other organisms. Amino acid sequences are numbered at the end of each sequence row. (*), (:) and (.) denote single fully conserved residues, residues with conserved strong groups and residues with conserved weak groups in Chyb, respectively. Aa, Adonis aestivalis; At, Arabidopsis thaliana; Bn, Brassica napus; Can, Capsicum annuum; Car, Coffea arabica; Cr, Chlamydomonas reinhardtii; Csi, Citrus sinensis; Csa, Crocus sativus; Cxm, Chrysanthemum x morifolium hybrid; Dc, Daucus carota; Dk, Diospyros kaki; Eo, Elaeis oleifera; Gl, Gentiana lutea; Gm, Glycine max; Hp, Haematococcus pluvialis; Lh, Lilium hybrid; Mz, Muriella zofingiensis; Sl, Solanum lycopersicum; Vv, Vitis vinifera; Zm, Zea mays. This alignment is produced by clustal 2.0.11 multiple sequence alignment program. 


\section{Conclusion:}

This study has annotated the salient features of randomly isolated EoChyb cDNA clone using Bioinformatics tools. Bioinformatics analyses revealed that EoChyb protein is carrying conserved domains for the beta-carotene hydroxylase and the sterol desaturase. Furthermore, the study also shows the 51 fully conserved amino acid residues in Chyb from the flowering (monocot and dicot) plants and algal members, which could be of relevance to gain insights into the evolution of Chyb.

\section{Acknowledgements}

Authors are grateful to the Ministry of Science, Technology and Innovation (MOSTI) of Malaysian Government for research funding [Grant Code: IRPA: 01-02-02-0014/PR0015/07-07], and to the United Plantation Berhad, Perak, Malaysia for supplying fruit samples of Elaeis oleifera for this study.

\section{References:}

[1] F Muniran et al. Indian J Exp Biol. 46: 79 (2008) [PMID: 18697576]

[2] O Asemota \& FH Shah, Afr J Biotechnol. 11: 595 (2004)

[3] BM Lange et al. PNAS. 97:2934-2939 (2000) [PMID: 10717007]

[4] J Ohlrogge, C Benning, Curr Opin Plant Biol. 3: 224 (2000) [PMID: 10837264]

[5] CM Ronning et al. Plant Physiol. 131:419 (2003) [PMID: 12586867]

[6] N Sreenivasulu et al. Plant J. 37:539 (2004) [PMID: 14756762]

[7] J Liu et al. Plant Mol Biol. 29: 685 (1995) [PMID: 8541495]

[8] CS Keith et al. Plant Physiol 101: 329 (1993) [PMID: 8278499]
[9] YS Park et al. Plant Physiol 103: 359 (1993) [PMID: 8029332]

[10] H Hofte et al. Plant J. 4: 1051 (1993) [PMID: 8281187]

[11] M Umeda \& H Uchimiya, Plant Physiol 106:1015(1994) [PMID: 12232382]

[12] A Marchler-Bauer et al. Nucleic Acids Res. 37: (D)205 (2009) [PMID: 18984618]

[13] JF Martin at al. Microb Cell Fact. 7: 3 (2008) [PMID: 18289382]

[14] I Baroli et al. Plant Cell. 15: 992 (2003) [PMID: 12671093]

[15] R Vallabhaneni et al. Plant Physiol 151: 1635 (2009) [PMID: 19767386]

[16] http://www.ncbi.nlm.nih.gov/

[17] http://www.justbio.com/

[18] http://www.currentprotocols.com/tools/dnarna-base-compositioncalculator

[19] http://align.genome.jp/

[20] FH Shah, TS Cha, Plant Sci. 154: 153 (2000) [PMID: 10729614]

[21] http://www.kazusa.or.jp/codon/cgibin/spsearch.cgi? species $=$ Elaeis $+\& \mathrm{c}=\mathrm{S}$

[22] R Singh et al. BMC Plant Biol. 9:114 (2009) [PMID: 19706196]

[23] BA Arthington, Gene. 102: 39 (1991) [PMID: 1864507]

[24] http://www.ncbi.nlm.nih.gov/taxonomy

[25] http://www.ncbi.nlm.nih.gov/Structure/cdd/cddsrv.cgi?ascbin=8\&ma xaln $=10$ \& seltype $=2 \&$ uid $=166242$

[26] http://www.ncbi.nlm.nih.gov/Structure/cdd/cddsrv.cgi?ascbin=8\&ma xaln $=10 \&$ seltype $=2 \&$ uid $=32818$

Edited by P. Kangueane

Citation: Bhore et al. Bioinformation 5(3): 104-112 (2010) License statement: This is an open-access article, which permits unrestricted use, distribution, and reproduction in any medium, for non-commercial purposes, provided the original author and source are credited. 


\section{Supplementary material:}

Supplementary Figure 1: Individual amino acids (\%) in the E. oleifera beta-carotene hydroxylase (Chyb) protein. On X axis, the amino acids are represented by a single letter code. The amino acid composition was calculated by ProtCalc program [17].

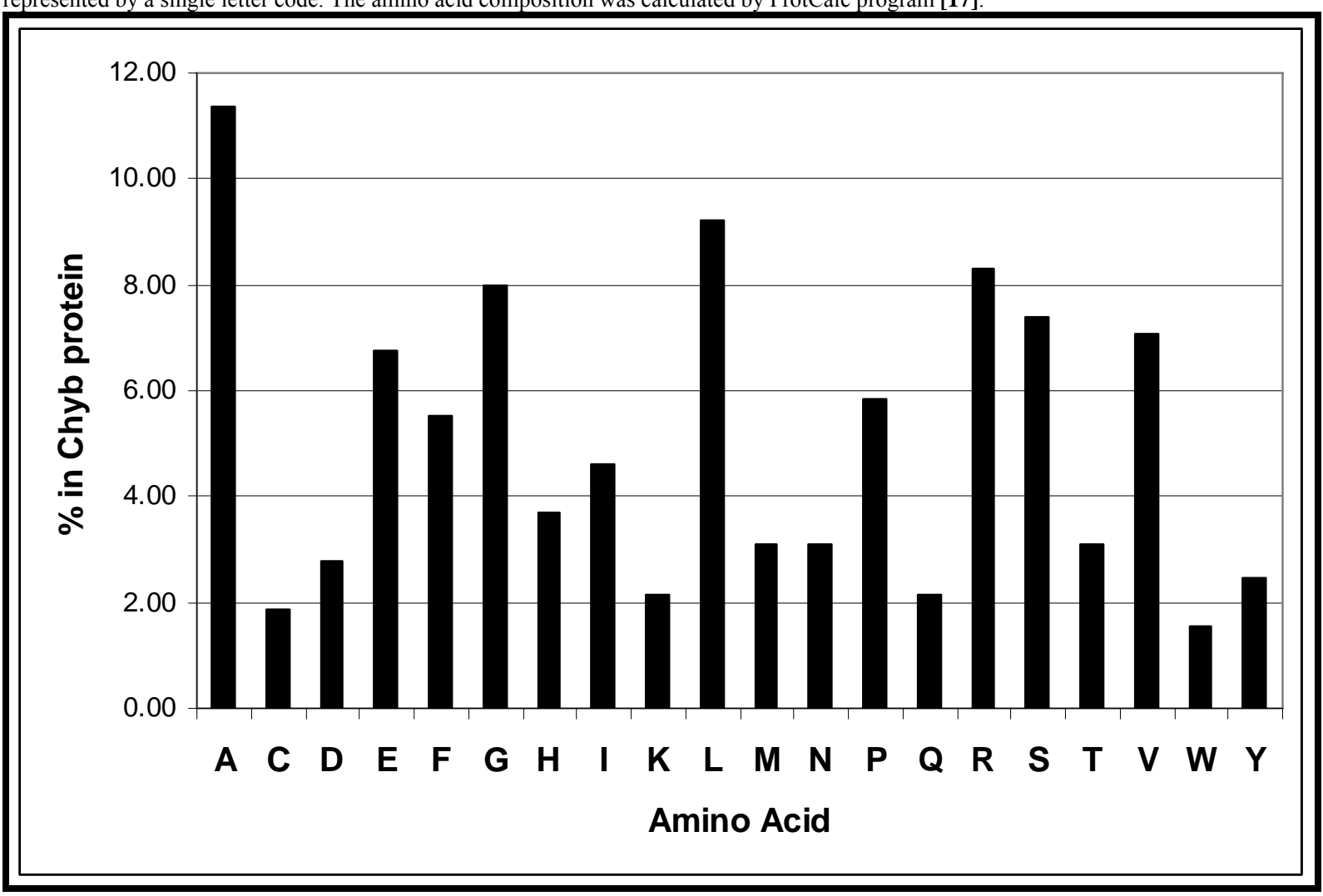

Table 1: The general features of Elaeis oleifera beta-carotene hydroxylase (EoChyb) cDNA and protein sequence General Features

cDNA sequence

Size, bp

Molecular Weight, Daltons

437125

5' UTR, bp

Coding Sequence

3' UTR, bp

Stop Codon

$\mathrm{G}+\mathrm{C}$ content, $\%$

Protein sequence

Length, amino acids 
Table 2: Comparison of the Elaeis oleifera beta-carotene hydroxylase (Chyb) with Chyb from monocots, dicots and algal members

\begin{tabular}{|c|c|c|c|c|c|}
\hline \multirow{2}{*}{ Species } & \multirow{2}{*}{$\begin{array}{c}\text { GenBank Accession } \\
\text { No. }\end{array}$} & \multicolumn{2}{|c|}{ Length compared } & \multicolumn{2}{|c|}{ Homology (\%) } \\
\hline & & nucleotide (bp) & $\begin{array}{c}\text { amino } \\
\text { acid }\end{array}$ & nucleotide level & $\begin{array}{c}\text { amino acid } \\
\text { level }\end{array}$ \\
\hline \multicolumn{6}{|l|}{ Monocots } \\
\hline Lilium hybrid & AB445122 & 722 & 177 & $85 \%$ & $97 \%$ \\
\hline Crocus sativus & AJ416711 & 918 & 305 & $81 \%$ & $82 \%$ \\
\hline Zea mays & AY844956 & 1392 & 308 & $87 \%$ & $98 \%$ \\
\hline \multicolumn{6}{|l|}{ Dicots } \\
\hline Daucus carota & DQ192193 & 1231 & 309 & $71 \%$ & $93 \%$ \\
\hline Arabidopsis thaliana & U58919 & 956 & 294 & $73 \%$ & $85 \%$ \\
\hline Brassica napus & EF026098 & 903 & 300 & $52 \%$ & $71 \%$ \\
\hline Chrysanthemum $x$ morifolium & AB205042 & 1227 & 136 & $74 \%$ & $97 \%$ \\
\hline Adonis aestivalis & EF120636 & 1187 & 309 & $74 \%$ & $82 \%$ \\
\hline Vitis vinifera & AF499108 & 900 & 299 & $78 \%$ & $98 \%$ \\
\hline Coffea arabica & DQ157169 & 933 & 310 & $75 \%$ & $92 \%$ \\
\hline Solanum lycopersicum & Y14809 & 1125 & 309 & $73 \%$ & $87 \%$ \\
\hline Gentiana lutea & $\mathrm{AB} 027187$ & 1519 & 320 & $73 \%$ & $82 \%$ \\
\hline Diospyros kaki & FJ790215 & 1365 & 312 & $79 \%$ & $90 \%$ \\
\hline Capsicuum annuum & Y09225 & 1112 & 315 & $71 \%$ & $96 \%$ \\
\hline Citrus sinensis & DQ228870 & 936 & 311 & $74 \%$ & $90 \%$ \\
\hline Glycine max & AY575953 & 1164 & 334 & $74 \%$ & $96 \%$ \\
\hline \multicolumn{6}{|l|}{ Algal members } \\
\hline Haematococcus pluvialis & DQ257289 & 906 & 298 & $34.8 \%$ & $81 \%$ \\
\hline Muriella zofingiensis & EU016205 & 1545 & 299 & $67 \%$ & $78 \%$ \\
\hline Chlamydomonas reinhardtii & XM_001698646 & 1757 & 297 & $72 \%$ & $84 \%$ \\
\hline
\end{tabular}




\section{Bioinformation}

Volume 5

open access

www.bioinformation.net

Issue 3

Hypothesis

Table 3: Amino acid composition of E. oleifera beta-carotene hydroxylase (Chyb) and in Chyb from other plant species and algal members

Source of Chyb and Their Amino Acid Composition (\%)

\begin{tabular}{|c|c|c|c|c|c|c|c|c|c|c|c|c|c|c|c|c|c|c|c|c|}
\hline \multirow{3}{*}{ Amino acid (single letter code) } & \multicolumn{20}{|c|}{ Source of Chyb and Their Amino Acid Composition (\%)" } \\
\hline & \multicolumn{13}{|c|}{ Dicotyledonous plants } & \multicolumn{4}{|c|}{ Monocotyledonous plants } & \multicolumn{3}{|c|}{ Algal Members } \\
\hline & Csi & Gm & Car & Sl & Gl & $\mathbf{D k}$ & Can & $\mathbf{V v}$ & Aa & Cxm & Bn & At & Dc & Eo & Lh & Csa & Zm & $\mathrm{Cr}$ & $\mathbf{M z}$ & Нр \\
\hline Alanine(A) & 9.97 & 9.88 & 11.61 & 10.03 & 8.12 & 10.58 & 11.43 & 8.36 & 10.36 & 7.35 & 8.67 & 8.50 & 11.33 & 11.38 & 9.60 & 12.13 & 16.56 & 14.14 & 11.04 & 13.76 \\
\hline Cysteine(C) & 0.96 & 0.90 & 0.97 & 0.65 & 0.94 & 0.96 & 1.59 & 1.00 & 0.97 & 0.74 & 1.00 & 0.68 & 0.97 & 1.85 & 0.56 & 0.33 & 0.65 & 0.67 & 2.01 & 1.01 \\
\hline Aspartic acid (D) & 1.61 & 1.80 & 1.94 & 1.94 & 2.81 & 2.56 & 2.86 & 2.34 & 1.94 & 2.21 & 3.33 & 2.72 & 2.27 & 2.77 & 2.26 & 1.97 & 1.95 & 3.03 & 2.68 & 2.35 \\
\hline Glutamic acid (E) & 7.72 & 6.29 & 7.42 & 7.77 & 6.88 & 7.69 & 7.30 & 7.36 & 7.44 & 7.35 & 6.67 & 7.48 & 7.44 & 6.77 & 7.91 & 7.54 & 6.17 & 6.40 & 4.68 & 5.03 \\
\hline Phenylalanine (F) & 8.04 & 6.29 & 7.10 & 7.77 & 6.56 & 6.73 & 7.94 & 4.68 & 7.12 & 6.62 & 6.67 & 6.80 & 6.80 & 5.54 & 8.47 & 6.56 & 5.84 & 6.40 & 5.35 & 3.69 \\
\hline Glycine (G) & 8.36 & 8.08 & 7.74 & 7.77 & 7.81 & 9.62 & 6.67 & 8.70 & 8.09 & 11.03 & 8.00 & 8.84 & 8.41 & 8.00 & 10.73 & 7.21 & 9.09 & 8.08 & 8.03 & 10.07 \\
\hline Histiaine(H) & 4.50 & 5.09 & 4.19 & 4.53 & 4.06 & 3.85 & 3.81 & 4.01 & 4.21 & 7.35 & 4.33 & 3.40 & 4.21 & 3.69 & 6.21 & 4.26 & 4.22 & 4.04 & 5.69 & 4.70 \\
\hline isoleucine(1) & 3.54 & 4.79 & 3.55 & 3.88 & 4.38 & 4.17 & 6.03 & 5.35 & 5.83 & 6.62 & 4.00 & 4.08 & 4.21 & 4.62 & 3.95 & 5.57 & 2.27 & 3.37 & 4.35 & 4.70 \\
\hline Lysine(K) & 5.79 & 3.89 & 5.16 & 5.83 & 5.94 & 4.17 & 5.40 & 3.68 & 3.88 & 7.35 & 4.33 & 5.10 & 4.53 & 2.15 & 3.39 & 3.28 & 2.27 & 4.38 & 3.34 & 3.02 \\
\hline Leucine(L) & 9.65 & 8.68 & 9.35 & 8.74 & 9.38 & 8.33 & 8.57 & 10.70 & 9.06 & 9.56 & 10.00 & 9.18 & 7.44 & 9.23 & 10.17 & 8.20 & 9.09 & 9.76 & 9.03 & 11.41 \\
\hline Methionine(M) & 3.22 & 3.29 & 3.23 & 3.24 & 3.12 & 2.88 & 3.17 & 4.35 & 4.85 & 3.68 & 3.33 & 3.06 & 3.24 & 3.08 & 3.95 & 2.95 & 3.57 & 4.71 & 3.68 & 3.36 \\
\hline Asparagine(N) & 1.93 & 2.10 & 1.94 & 1.94 & 2.50 & 1.28 & 2.22 & 3.01 & 4.21 & 3.68 & 2.33 & 3.06 & 2.91 & 3.08 & 3.39 & 1.97 & 1.3 & 1.68 & 2.01 & 1.01 \\
\hline Prome(P) & 5.47 & 5.99 & 4.19 & 5.50 & 5.00 & 5.77 & 5.40 & 4.68 & 4.86 & 5.88 & 5.00 & 4.42 & 4.85 & 5.85 & 5.65 & 6.23 & 7.14 & 6.40 & 5.69 & 4.70 \\
\hline Glutamine(Q) & 1.93 & 2.40 & 1.94 & 0.97 & 1.88 & 1.28 & 1.90 & 1.00 & 1.62 & 2.21 & 1.33 & 1.02 & 0.97 & 2.15 & 0.56 & 0.98 & 0.65 & 3.37 & 4.35 & 3.36 \\
\hline Arginine(R) & 5.14 & 6.29 & 7.74 & 5.50 & 5.31 & 6.09 & 6.03 & 6.02 & 7.12 & 3.68 & 6.67 & 6.12 & 6.47 & 8.31 & 5.65 & 9.18 & 9.09 & 5.05 & 5.02 & 7.38 \\
\hline Serine(S) & 5.47 & 8.38 & 5.81 & 7.77 & 9.38 & 7.37 & 6.67 & 10.37 & 5.50 & 2.21 & 9.67 & 11.56 & 9.06 & 7.38 & 3.39 & 6.56 & 3.9 & 3.37 & 7.36 & 4.36 \\
\hline Threonine(T) & 4.82 & 3.29 & 4.19 & 4.85 & 4.06 & 4.17 & 2.86 & 3.01 & 3.56 & 1.47 & 3.67 & 2.72 & 2.27 & 3.08 & 1.69 & 4.59 & 3.9 & 3.37 & 4.68 & 4.70 \\
\hline & 7.72 & 7.78 & 8.06 & 7.12 & 8.12 & 8.01 & 5.71 & 7.02 & 5.83 & 7.35 & 7.33 & 6.12 & 7.77 & 7.08 & 7.91 & 6.56 & 8.44 & 8.08 & 6.69 & 6.71 \\
\hline Tryptophan(W) & 1.93 & 2.10 & 1.61 & 1.62 & 1.56 & 1.60 & 1.59 & 1.67 & 1.62 & 0.74 & 1.67 & 1.70 & 1.94 & 1.54 & 2.26 & 1.64 & 1.62 & 1.01 & 2.01 & 2.01 \\
\hline Tyrosine(Y) & 2.25 & 2.69 & 2.26 & 2.59 & 2.19 & 2.88 & 2.86 & 2.68 & 1.94 & 2.94 & 3.00 & 3.40 & 2.91 & 2.46 & 2.26 & 2.30 & 2.27 & 2.69 & 2.34 & 2.68 \\
\hline
\end{tabular}




\section{Bioinformation}

www.bioinformation.net

\section{Volume 5}

Issue 3

\section{Hypothesis}

"Source of Chyb: Aa, Adonis aestivalis; At, Arabidopsis thaliana; Bn, Brassica napus; Can, Capsicum annuum; Car, Coffea arabica; Cr, Chlamydomonas reinhardtii; Csi, Citrus sinensis; Csa, Crocus sativus; Cxm, Chrysanthemum x morifolium hybrid; Dc, Daucus carota; Dk, Diospyros kaki; Eo, Elaeis oleifera; Gl, Gentiana lutea; Gm, Glycine max; Hp, Haematococcus pluvialis; Lh, Lilium hybrid; Mz, Muriella zofingiensis; Sl, Solanum lycopersicum; Vv, Vitis vinifera; Zm, Zea mays. The amino acid composition was calculated by ProtCalc program [17]. 